\title{
Sulforaphane induces apoptosis in T24 human urinary bladder cancer cells through a reactive oxygen species-mediated mitochondrial pathway: The involvement of endoplasmic reticulum stress and the Nrf2 signaling pathway
}

\author{
GUK HEUI JO ${ }^{1}$, GI-YOUNG KIM ${ }^{2}$, WUN-JAE KIM ${ }^{3}$, KUN YOUNG PARK ${ }^{4}$ and YUNG HYUN CHOI ${ }^{1,5}$ \\ ${ }^{1}$ Department of Biochemistry, Dongeui University College of Korean Medicine, Busan 614-052; \\ ${ }^{2}$ Laboratory of Immunobiology, Department of Marine Life Sciences, Jeju National University, \\ Jeju 690-756; ${ }^{3}$ Department of Urology, Chungbuk National University College of Medicine, \\ Cheongju 361-763; ${ }^{4}$ Department of Food and Nutrition, College of Human Ecology, \\ Busan National University, Busan 609-735; ${ }^{5}$ Anti-Aging Research Center and \\ Blue-Bio Industry Regional Innovation Center, Dongeui University, \\ Busan 614-714, Republic of Korea
}

Received April 25, 2014; Accepted June 19, 2014

DOI: $10.3892 /$ ijo.2014.2536

\begin{abstract}
Sulforaphane, a naturally occurring isothiocyanate found in cruciferous vegetables, has received a great deal of attention because of its ability to inhibit cell proliferation and induce apoptosis in cancer cells. In this study, we investigated the anticancer activity of sulforaphane in the T24 human bladder cancer line, and explored its molecular mechanism of action. Our results showed that treatment with sulforaphane inhibited cell viability and induced apoptosis in T24 cells in a concentration-dependent manner. Sulforaphane-induced apoptosis was associated with mitochondria dysfunction, cytochrome $c$ release and Bcl-2/Bax dysregulation. Furthermore, the increased activity of caspase- 9 and -3 , but not caspase- 8 , was accompanied by the cleavage of poly ADP-ribose polymerase, indicating the involvement of the mitochondria-mediated intrinsic apoptotic pathway. Concomitant with these changes, sulforaphane triggered reactive oxygen species (ROS) generation, which, along with the blockage of sulforaphane-induced loss of mitochondrial membrane potential and apoptosis, was strongly attenuated by the ROS scavenger N-acetyl-L-cysteine. Furthermore, sulforaphane was observed to activate endoplasmic reticulum (ER) stress and the nuclear factor-E2-related factor-2 (Nrf2)
\end{abstract}

Correspondence to: Dr Yung Hyun Choi, Department of Biochemistry, Dongeui University College of Korean Medicine, Yangjeong-dong San 45, Busanjin-gu, Busan 614-052, Republic of Korea

E-mail: choiyh@deu.ac.kr

Key words: sulforaphane, apoptosis, reactive oxygen species, endoplasmic reticulum stress, nuclear factor-E2-related factor-2 signaling pathway, as demonstrated by the upregulation of ER stress-related proteins, including glucose-regulated protein 78 and C/EBP-homologous protein, and the accumulation of phosphorylated Nrf2 proteins in the nucleus and induction of heme oxygenase-1 expression, respectively. Taken together, these results demonstrate that sulforaphane has antitumor effects against bladder cancer cells through an ROS-mediated intrinsic apoptotic pathway, and suggest that ER stress and Nrf2 may represent strategic targets for sulforaphane-induced apoptosis.

\section{Introduction}

Bladder cancer is the most common malignancy of the urinary tract, with an incidence four times higher in men than in women, and has a high rate of tumor recurrence. Because the incidence of urinary bladder cancer has continuously increased over the past two decades, bladder cancer is clearly recognized as a significant public health issue around the world, especially in industrialized countries $(1,2)$. Bladder cancers can be divided into two major subgroups that possess distinct clinical, pathological and molecular characteristics. More than $70 \%$ of bladder cancer patients have non-muscle invasive papillary tumors that are managed with transurethral resection of the tumors followed by intravesical instillation of anticancer agents, and such patients have a good prognosis. However, the other $30 \%$, who have muscle-invasive tumors, have a very poor prognosis, and these tumors can rapidly progress to become metastatic and can lead to death. Although current treatments for bladder cancer-surgery, radiation therapy, chemotherapy or their combination-prolong survival time, bladder cancer tends to recur and progress $(3,4)$. Therefore, efforts to develop a novel treatment to combat the disease with complete efficacy and low toxicity must necessarily be increased. 
Apoptosis, a form of genetically programmed cell death, plays an important role in the cellular response to genotoxic stress; hence, loss of apoptotic response in tumor cells represents an effective mechanism of malignant progression and resistance to treatment $(5,6)$. Therefore, searching for agents that can trigger tumor cell apoptosis has become an attractive strategy in the discovery of anticancer drugs. Cells undergoing apoptosis are characterized by membrane blebbing, cytoplasmic shrinkage, DNA fragmentation and apoptotic body formation. There are two main apoptotic pathways in mammals: the extrinsic or death receptor-mediated pathway and the intrinsic or mitochondria-mediated pathway. The former is triggered by engagement of cell-surface death receptors of the tumor necrosis factor receptor family with their ligands, leading to the cleavage of caspase- 8 . Whereas the latter is activated following mitochondrial depolarization, release of cytochrome $c$ and the subsequent activation of caspase-9; the event regulated by interactions between proteins related to Bcl-2 family $(7,8)$. Although low physiological levels of reactive oxygen species (ROS) serve as a signaling messenger to mediate various biological responses, excessive intracellular ROS, which can induce depolarization of the mitochondrial membrane potential (MMP, $\Delta \Psi \mathrm{m}$ ), are also considered an apoptotic death signal that ultimately activates the intrinsic apoptotic pathway. Moreover, cancer cells are more sensitive to fluctuations in ROS levels than normal cells; therefore, ROS are considered an important target in anticancer agent research (9-11).

Phytochemicals are regarded as a precious alternative to modern medicine, and investigations of active components with anticancer potential and fewer side effects have opened up new research avenues (12-14). Among such phytochemicals, sulforaphane (4-methylsulfinylbutyl isothiocyanate) has been identified as a non-toxic isothiocyanate of organosulfur compounds that is found in cruciferous vegetables such as broccoli, Brussels sprouts and cabbage. A body of evidence shows that this phytochemical is able to inhibit the growth of and induce apoptosis in many different human cancer cells (15-17). In the case of bladder cancers, sulforaphane has also shown anti-bladder cancer activity, the reported mechanisms of which include modulation of cyclooxygenase-2 expression associated with $\mathrm{p} 38$ mitogen-activated protein kinase activation in T24 bladder cancer cells (18), inhibition of 4-aminobiphenyl-induced DNA damage in RT4 bladder cancer cells and in mouse bladder tissue (19) and inhibition of invasion and metastasis by suppressing the epithelialto-mesenchymal transition process (20). We also recently reported that sulforaphane-induced growth inhibition was associated with a mitotic arrest and apoptosis of 5637 bladder cancer cells via ROS-dependent pathway (21). However, the precise cellular mechanisms of sulforaphane's effect on bladder cancer cells are not completely understood. Therefore, in the present study, we used the T24 human bladder cancer cell line to further examine the molecular events responsible for sulforaphane-induced apoptosis. Sulforaphane exhibited significant growth inhibitory effects and an increase in intrinsic apoptotic cell death, leading to the regulation of Bcl-2 family proteins. Furthermore, our data provided strong evidence that ROS generation was a crucial event in the apoptotic response to sulforaphane in T24 cells.

\section{Materials and methods}

Reagents. Sulforaphane was purchased from Sigma-Aldrich Chemical Co. (St. Paul, MN), dissolved in dimethyl sulfoxide (DMSO, Sigma-Aldrich), and then diluted with the medium to the desired concentration prior to use. RPMI-1640 medium and fetal bovine serum (FBS) were obtained from Gibco-BRL (Gaithersburg, MD). 3-(4,5-Dimetylthiazol-2-yl)-2,5-diphenyltetrazolium (MTT), 4,6-diamidino-2-phenylindole (DAPI), propidium iodide (PI), 2,7-dichlorodihydrofluorescein diacetate (DCFH-DA), 5,5',6,6'-tetrachloro-1,1',3,3'-tetraethylimidacarbocyanine iodide (JC-1) and $\mathrm{N}$-acetyl L-cysteine (NAC) were obtained from Sigma-Aldrich. Caspase activity assay kits and an enhanced chemiluminescence (ECL) kit were purchased from R\&D Systems (Minneapolis, MN) and Amersham Life Science Corp. (Arlington Heights, IL), respectively. Primary antibodies were purchased from Santa Cruz Biotechnology (Santa Cruz, CA), Chemicon (Temecula, CA), and Abcam (Cambridge, UK). Peroxidase-labeled donkey antirabbit and sheep anti-mouse immunoglobulin were purchased from Amersham Life Science Corp. All other chemicals were purchased from Sigma-Aldrich.

Cell culture and cytotoxicity assay. T24 cells were purchased from American Type Culture Collection (Manassas, VA) and maintained in RPMI-1640 medium supplemented with $10 \%$ FBS, $2 \mu \mathrm{m} \mathrm{L}$-glutamine and penicillin/streptomycin under a humidified condition of $5 \% \mathrm{CO}_{2}$ at $37^{\circ} \mathrm{C}$. In order to measure the inhibition of $\mathrm{T} 24$ cell proliferation by sulforaphane, cells were plated in 6 -well culture plates $\left(1 \times 10^{5}\right.$ cells/well $)$ and allowed to adhere overnight, and were then treated with different concentrations of sulforaphane for $24 \mathrm{~h}$. After treatment, cells were incubated with MTT solution $(5 \mathrm{mg} / \mathrm{ml})$ for $3 \mathrm{~h}$, and the medium was then removed. The formazan precipitate was dissolved in DMSO, and the absorbance was read at $540 \mathrm{~nm}$ in an ELISA reader (Dynatech Laboratories, Chantilly, VA). Morphological changes were monitored by obtaining photomicrographs under an inverted phase contrast microscope (Carl Zeiss, Oberkochen, Germany).

Morphological observation of nuclear change. After culture with various concentrations of sulforaphane for $24 \mathrm{~h}$, cells were washed with phosphate-buffered saline (PBS) and fixed with $3.7 \%$ paraformaldehyde in PBS for $10 \mathrm{~min}$ at room temperature. Fixed cells were washed with PBS, and stained with DAPI solution for $10 \mathrm{~min}$ at room temperature. Then, the nuclear morphology of the cells was examined using a fluorescence microscope (Carl Zeiss).

Measurement of DNA fragmentation. Cells were harvested, washed with PBS and lysed in a buffer [50 mM Tris ( $\mathrm{pH} 8.0)$, $0.5 \%$ sarkosyl, $0.5 \mathrm{mg} / \mathrm{ml}$ proteinase $\mathrm{K}$ and $1 \mathrm{mMEDTA}]$ at $55^{\circ} \mathrm{C}$ for $3 \mathrm{~h}$. Lysates were then treated with RNase A $(0.5 \mathrm{mg} / \mathrm{ml})$ for $2 \mathrm{~h}$ at $56^{\circ} \mathrm{C}$. The lysates were centrifuged at $10,000 \mathrm{~g}$ for $20 \mathrm{~min}$. The genomic DNA in the supernatant was extracted with an equal volume of neutral phenol/chloroform/isoamyl alcohol mixture (25/24/1). Approximately $20 \mu \mathrm{g}$ of DNA was loaded into each well of a $1.5 \%$ agarose gel and separated by electrophoresis at $50 \mathrm{~V}$ for $120 \mathrm{~min}$ in Tris-borate/EDTA electrophoresis buffer (TBE). The DNA was visualized and 
photographed under ultraviolet (UV) illumination after staining with $0.1 \mu \mathrm{g} / \mathrm{ml}$ ethidium bromide (EtBr).

Flow cytometric analysis of DNA content for apoptosis. After treatment with sulforaphane for $24 \mathrm{~h}$, cells were harvested, washed with $\mathrm{PBS}$ and fixed with $70 \%$ ethanol at $-20^{\circ} \mathrm{C}$ overnight. Cells were washed twice, resuspended in PBS containing $40 \mu \mathrm{g} / \mathrm{ml} \mathrm{PI,} 0.1 \mathrm{mg} / \mathrm{ml}$ RNase A and $0.1 \%$ Triton $\mathrm{X}-100$ in a dark room for $30 \mathrm{~min}$ at $37^{\circ} \mathrm{C}$, and analyzed by flow cytometry (Becton-Dickinson, San Jose, CA). The cell cycle distribution and sub-G1 population (apoptosis) were determined and analyzed.

Western blot analysis. For isolation of total protein fractions, cells were collected, washed twice with cold PBS, and lysed with cell lysis buffer [20 mM Tris pH 7.5, $150 \mathrm{mM} \mathrm{NaCl}, 1 \%$ Triton X-100, $2.5 \mathrm{mM}$ sodium pyrophosphate, $1 \mathrm{mM}$ EDTA, $0.5 \mathrm{~g} / \mathrm{ml}$ leupeptin, $1 \% \mathrm{Na}_{3} \mathrm{CO}_{4}, 1 \mathrm{mM}$ phenylmethane-sulfonyl fluoride]. The cell lysates were centrifuged at $13,000 \mathrm{~g}$ at $4^{\circ} \mathrm{C}$, and the supernatant was collected for western blot analysis, and measured for protein concentration by using the Bio-Rad protein assay kit (Bio-Rad Laboratories, Hercules, CA). In a parallel experiment, mitochondrial and cytosol cellular fractions were prepared using a Cytosol/Mitochondria Fractionation kit (Calbiochem, San Diego, CA) according to the manufacturer's protocol. The equal aliquots containing 30-50 $\mu \mathrm{g}$ of each lane were separated by sodium dodecyl sulfate (SDS)-polyacrylamide gel electrophoresis followed by electro-transfer onto nitrocellulose membranes (Schleicher \& Schuell, Keene, NH). The membranes were incubated overnight at $4^{\circ} \mathrm{C}$ with the primary antibodies and the corresponding horseradish peroxidase-conjugated secondary antibodies. The protein bands were visualized using an ECL kit.

Caspase activity assay. Activities of caspases were determined by use of colorimetric assay kits, which utilize synthetic tetrapeptides [Asp-Glu-Val-Asp (DEAD) for caspase-3, Ile-GluThr-Asp (IETD) for caspase-8 and Leu-Glu-His-Asp (LEHD) for caspase-9] labeled with p-nitroaniline (pNA). Briefly, sulforaphane-treated and untreated cells were lysed in the supplied lysis buffer. Supernatants were collected and incubated with the supplied reaction buffer containing dithiothreitol (DTT) and DEAD-pNA, IETD-pNA or LEHD-pNA as substrates at $37^{\circ} \mathrm{C}$. The reactions were measured by changes in absorbance at $405 \mathrm{~nm}$ using an ELISA reader.

Flow cytometric determination of the cellular redox state by ROS. The intracellular accumulation of ROS was examined by flow cytometry after being stained with the cell permeable fluorescent probe, DCFH-DA. Briefly, the cells were washed, resuspended in PBS and incubated with $10 \mu \mathrm{M}$ DCFH-DA for $20 \mathrm{~min}$ at $37^{\circ} \mathrm{C}$ in the dark. The ROS production in the cells was monitored with a flow cytometer (22).

Flow cytometric detection of MMP $(\Delta \Psi m)$. The values of MMP were determined using the dual-emission potentialsensitive probe JC-1. Briefly, cells were collected and resuspended in PBS supplemented with $10 \mu \mathrm{M}$ JC-1 for $30 \mathrm{~min}$ at $37^{\circ} \mathrm{C}$ in the dark. After the $\mathrm{JC}-1$ was removed, the cells were washed with PBS to remove unbound dye, and the

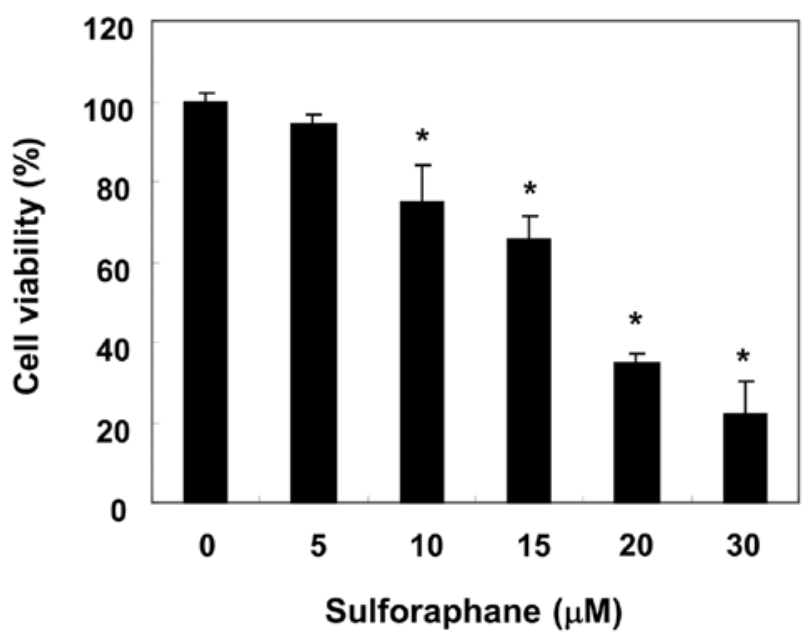

Figure 1. Inhibition of cell viability by sulforaphane in T24 cells. Cells were seeded at $2 \times 10^{5}$ cells $/ \mathrm{ml}$ and were then treated with the indicated concentrations of sulforaphane for $24 \mathrm{~h}$. Cell viability was determined by MTT assay. The data are expressed as the mean \pm SD of three independent experiments $\left({ }^{*} \mathrm{p}<0.05\right.$ vs. untreated control)

amount of JC-1 retained by cells was immediately analyzed by flow cytometry.

Statistical analysis. Unless otherwise stated, data are expressed as means \pm standard deviation (SD) and analyzed statistically by one-way analysis of variance. A value of $\mathrm{p}<0.05$ was considered statistically significant.

\section{Results}

Sulforaphane treatment reduces cell viability and induces apoptosis in T24 cells. Initially, the effects of sulforaphane on proliferation of T24 cells were measured by an MTT assay. As shown in Fig. 1, sulforaphane treatment inhibited the cell viability of $\mathrm{T} 24$ cells in a concentration-dependent manner: sulforaphane at 10 and $20 \mu \mathrm{M}$ for $24 \mathrm{~h}$ inhibited cell proliferation by 25 and $62 \%$, respectively. In addition, sulforaphane stimulation significantly induced morphological changes, including extensive cytosolic vacuolization and the appearance of irregular cell membrane buds (Fig. 2A). To examine whether the cytotoxicity of sulforaphane was due primarily to apoptosis, we assessed the apoptosis parameters of T24 cells in response to sulforaphane treatment. As shown in Fig. 2B, the nuclear structure of the control cells remained intact, whereas nuclear chromatin condensation and fragmentation, both of which are characteristics of apoptosis, were increased in a concentrationdependent manner in cells treated with sulforaphane, which was associated with increased DNA fragmentation (Fig. 2C). Under the same conditions, results of flow cytometry further demonstrated that treatment with sulforaphane induced a concentration-dependent accumulation of apoptotic sub-G1 population of T24 cells (Fig. 1D). These results indicate that the inhibition of cell viability observed in response to sulforaphane was associated with the induction of apoptosis.

Sulforaphane treatment activates caspase-3 and -9 in T24 cells. Several gene products are known to be important in 


\section{Sulforaphane $(\mu \mathrm{M})$}

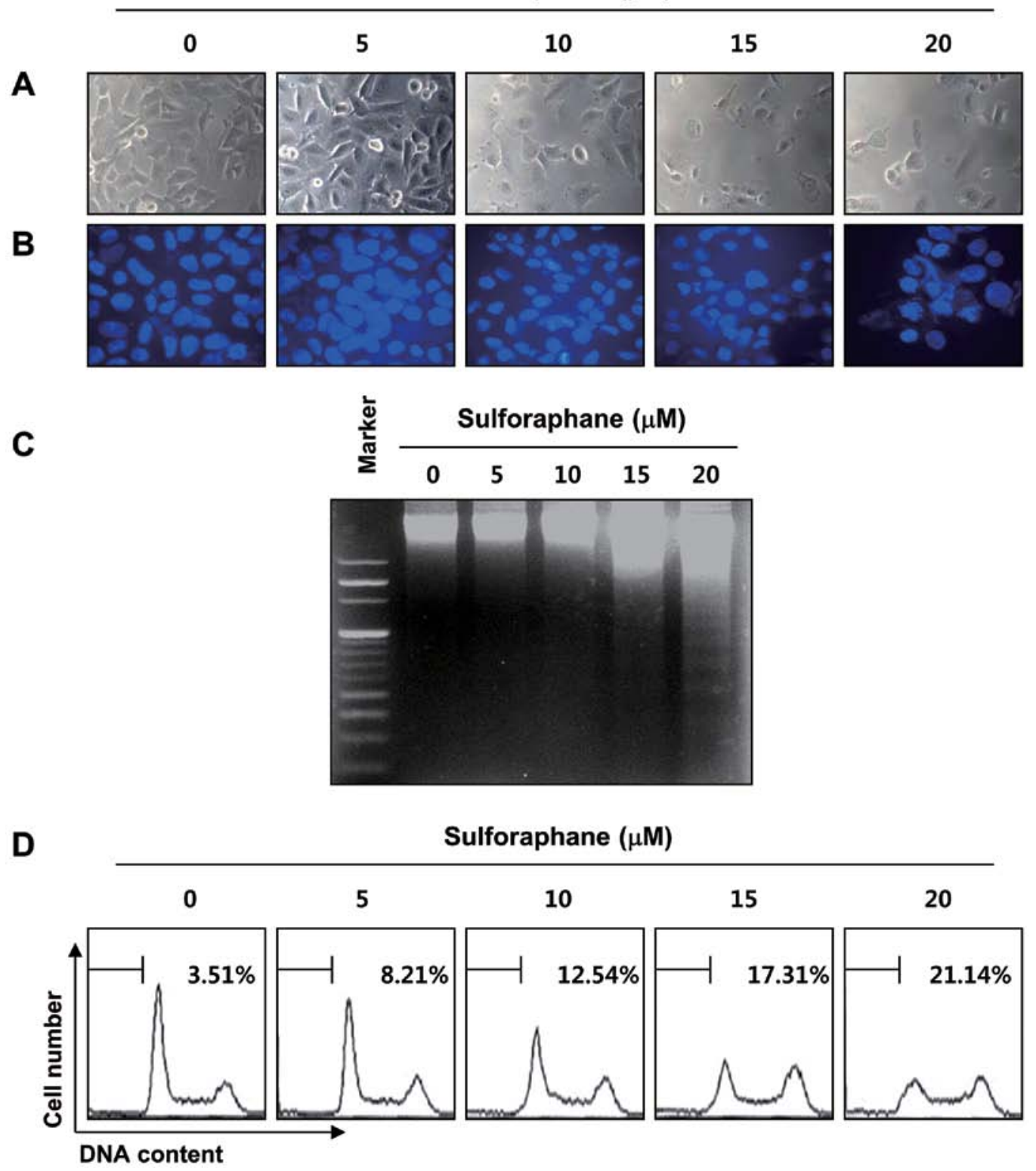

Figure 2. Induction of apoptosis by sulforaphane in T24 cells. (A) The cells were treated with the indicated concentrations of sulforaphane for 24 h and then sampled, and the changes in cell morphology were observed. (B) The cells were fixed and stained with DAPI solution, and the stained nuclei were then observed under a fluorescent microscope (original magnification, x400). (C) For analysis of DNA fragmentation, genomic DNA was extracted, electrophoresed in a $1.5 \%$ agarose gel and visualized by EtBr staining. (D) To quantify the degree of apoptosis induced by sulforaphane, cells were evaluated by a flow cytometer for sub-G1 DNA content, which represents the cells undergoing apoptotic DNA degradation. The data are expressed as the mean \pm SD of three independent experiments.

controlling the apoptotic process. Among them, caspases, a family of cysteine proteases, play essential roles as important mediators in apoptosis and as determinants of general apoptotic morphology through the cleavage of various cellular substrates, including poly ADP-ribose polymerase (PARP), an endogenous substrate of activated caspase-3 $(23,24)$. To elucidate the molecular mechanisms of sulforaphane-induced apoptosis, we assessed whether sulforaphane induces the proteolytic processing of caspases. As illustrated in Fig. 3A, we detected a concentration-dependent upregulation of cleaved caspase- 3 and -9 , whereas the cleaved forms of caspase- 8 were not detected. In addition, to quantify the proteolytic activation of the caspases, we evaluated in vitro caspase activities using fluorogenic substrates (Fig. 3B). Our results indicated that treatment with sulforaphane significantly increased the activities of caspase- 3 and -9 compared with control cells, whereas a very low level of caspase- 8 activity was detected after the sulforaphane treatment, suggesting the likely involvement of a mitochondria-dependent cascade for caspase activation. To further identify the activation of the caspase cascade, the levels of PARP were examined by western blot analysis. As shown in Fig. 3A, sulforaphane induced a concentration-dependent proteolytic cleavage of PARP, resulting in a reduction of the $116-\mathrm{kDa}$ protein and accumulation of the $85-\mathrm{kDa}$ fragment. In addition, the levels of inhibitor of apoptosis proteins (IAP) family members, including XIAP, cIAP-1 and cIAP-2, were 
A

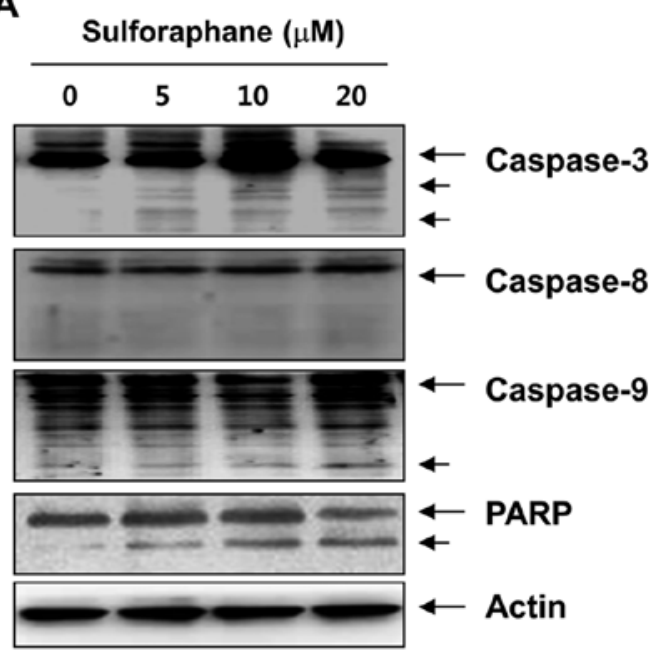

\section{Sulforaphane $(\mu \mathrm{M})$}

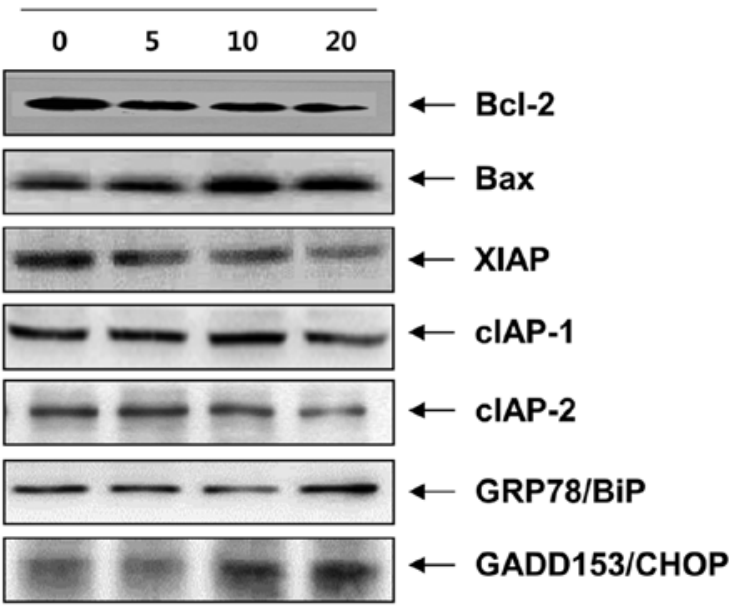

B

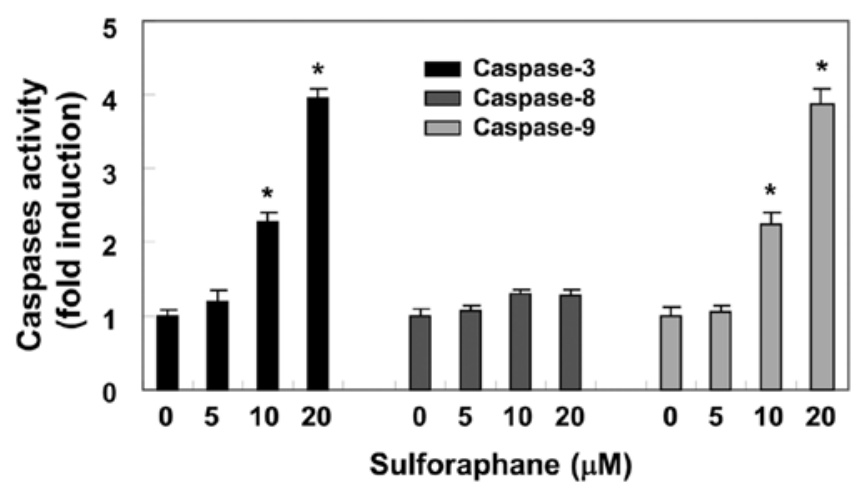

Figure 3. Effects of sulforaphane treatment on the levels of apoptosis-related and ER stress-related proteins in T24 cells. (A) Cells were treated with the indicated concentrations of sulforaphane for $24 \mathrm{~h}$. The cells were lysed, and the cellular proteins were then separated by SDS-polyacrylamide gels and transferred onto nitrocellulose membranes. The proteins were visualized using the indicated antibodies and an ECL detection system. Actin was used as an internal control. (B) After $24 \mathrm{~h}$ of incubation with the indicated concentrations of sulforaphane, the cells were lysed and aliquots were assayed for in vitro caspase-3, -8 and -9 activity using DEVD-pNA, IETD-pNA and LEHD-pNA as substrates, respectively, at $37^{\circ} \mathrm{C}$ for $1 \mathrm{~h}$. The released fluorescent products were measured. Each point represents the mean \pm SD of three independent experiments ( $(\mathrm{p}<0.05$ vs. untreated control).

inhibited by sulforaphane treatment in a concentration-dependent manner (Fig. 3A).

Sulforaphane-induced apoptosis involves alterations in the intracellular distribution of Bcl-2 family proteins and cytochrome $c$ in $T 24$ cells. It has been recognized that the Bcl-2 family members play crucial roles in regulating apoptosis by functioning as promoters (e.g., Bax) or inhibitors (e.g., Bcl-2) of cell death. Mitochondria are specialized organelles containing an outer membrane and an inner membrane separated by an intermembrane space that contains many proapoptotic proteins, such as cytochrome $c(25,26)$. We therefore examined the expression of these molecules after sulforaphane treatment. Western blot analysis data showed that sulforaphane markedly increased protein levels of proapoptotic Bax. In contrast to Bax, anti-apoptotic Bcl-2 expression decreased mildly following sulforaphane treatment. To further characterize the apoptotic effect of sulforaphane in T24 cells, we analyzed the translocation of Bax and the release of cytochrome $c$ using cytosol and mitochondria fractionation. Notably, as shown in Fig. 4, sulforaphane treatment led to a concentration-dependent increase in cytosolic cytochrome $c$ levels and triggered the

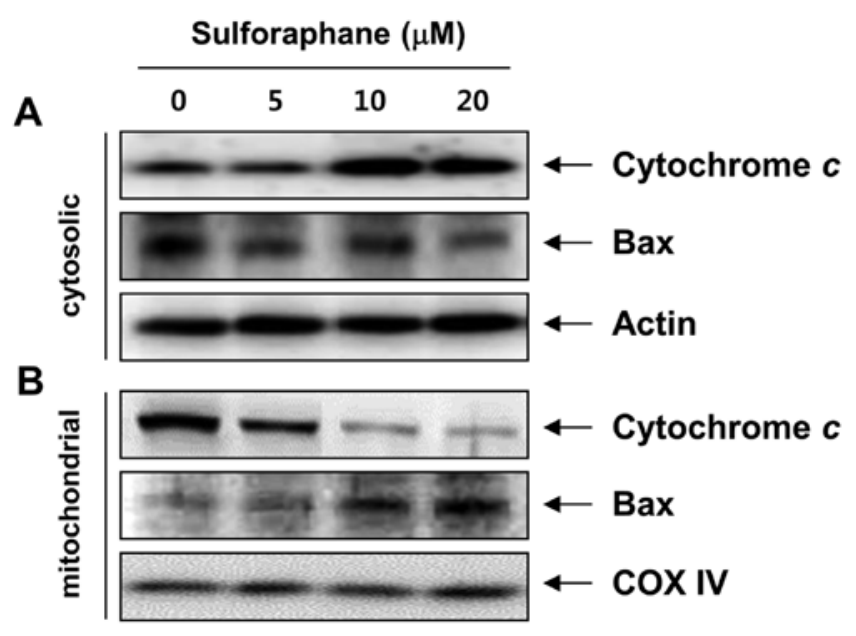

Figure 4. Translocation of Bax and release of cytochrome $c$ by sulforaphane in T24 cells. Cells were treated with the indicated concentrations of sulforaphane for $24 \mathrm{~h}$. The cytosolic (A) and mitochondrial (B) proteins were extracted and analyzed by western blotting using anti-cytochrome $c$ and anti-Bax antibodies. Actin and cytochrome oxidase IV (COX IV) were used as internal controls for the cytosolic and mitochondrial fractions, respectively. 


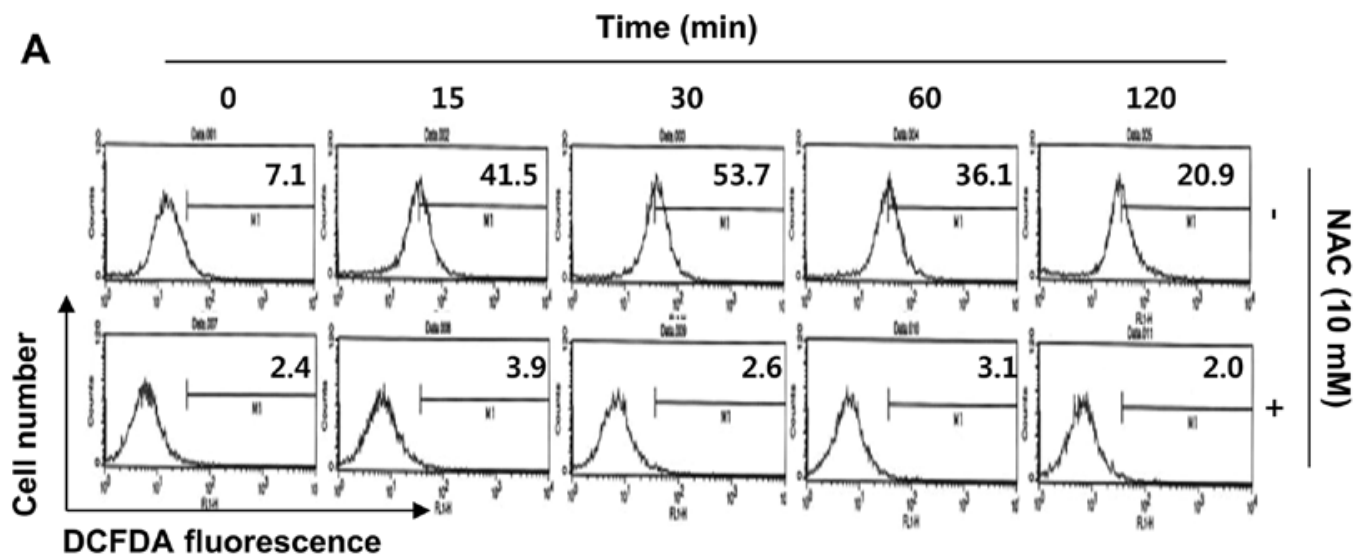

B

Time (h)

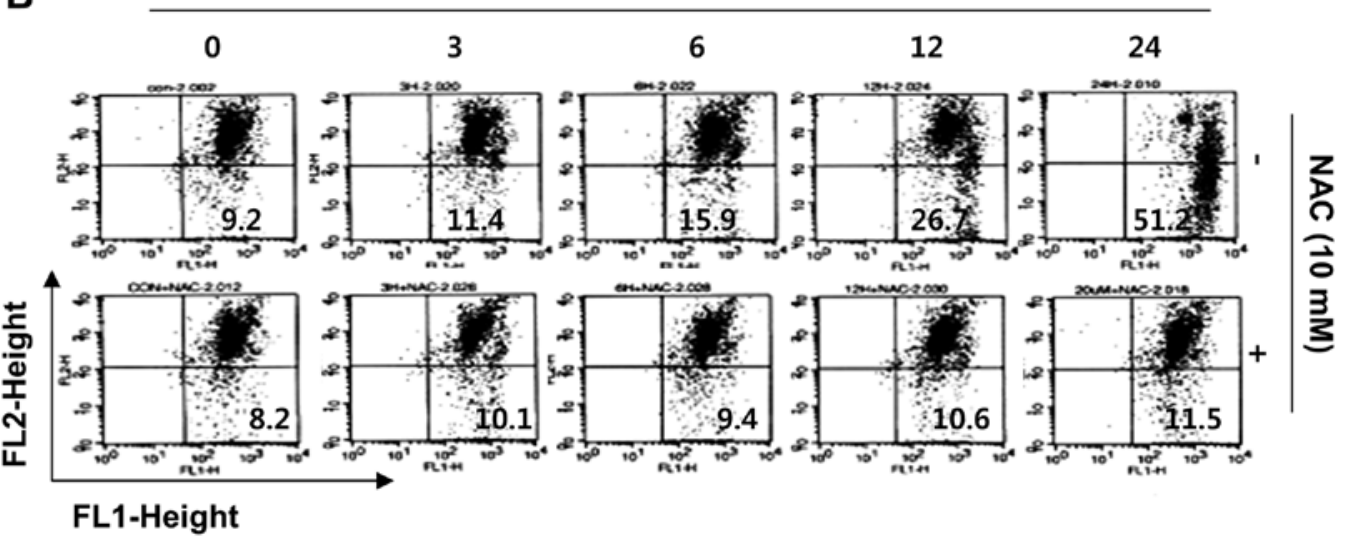

Figure 5. Effects of sulforaphane on the MMP values and ROS generation in T24 cells. (A) Cells were pretreated with or without $10 \mathrm{mM} \mathrm{NAC} \mathrm{for} 1 \mathrm{~h}$ before treatment with $20 \mu \mathrm{M}$ sulforaphane for the indicated times. The cells were collected, incubated with $20 \mathrm{mM} \mathrm{DCFH}-\mathrm{DA}$ at $37^{\circ} \mathrm{C}$ for 30 min, and ROS generation was then measured using a flow cytometer. (B) Cells were pretreated with $10 \mathrm{mM} \mathrm{NAC} 1 \mathrm{~h}$ prior to $20 \mu \mathrm{M}$ sulforaphane treatment for $24 \mathrm{~h}$. The cells were incubated with $10 \mu \mathrm{M} \mathrm{JC}-1$ for $30 \mathrm{~min}$ at $37^{\circ} \mathrm{C}$ in the dark, and then analyzed by a flow cytometer. The results are presented as the mean of two independent experiments.

translocation of Bax to the mitochondria. In addition, the MMP, which is regulated by the Bcl-2 family, was gradually reduced in the sulforaphane-treated cells compared to the control cells (Fig. 5B). These results suggest that sulforaphane-induced apoptosis, which took place by promoting the translocation of Bax to mitochondria, followed by a loss of MMP and release of cytochrome $c$ into the cytosol, results in the activation of caspase- 9 and -3 , thus leading to apoptosis in the cells. These observations also suggest that sulforaphane induced apoptosis in T24 cells via the mitochondrial pathway.

Sulforaphane induces the activation of ER stress pathway in T24 cells. Evidence has accumulated from many studies that apoptosis associated with endoplasmic reticulum (ER) stress may be responsible for cell death induced by antitumor agents $(27,28)$. ER stress can be characterized by an increase in ER stress-associated molecules. In particular, glucose-regulated protein (GRP) 78 and $\mathrm{C} / \mathrm{EBP}$-homologous protein (CHOP) have been considered as vital proteins of ER stress response. To examine whether ER stress was involved in sulforaphane-induced apoptosis, we followed the behavior of these two stress markers in response to sulforaphane treatment. Our data revealed that sulforaphane exposure increased the expression of GRP78 as well as CHOP compared with control (Fig. 4A) in a concentration-dependent manner. These results show that ER stress was involved in sulforaphane-induced apoptosis in T24 cells, as evidenced by upregulation of the expression of ER stress-associated proteins.

Generation of ROS is required as a mediator for sulforaphaneinduced mitochondrial dysfunction in R24 cells. Because oxidative stress and ROS generation are directly involved in protease cascades, such as induction of caspases during apoptosis regulation $(9,10)$, we investigated whether the elevated generation of ROS production is related to sulforaphaneinduced apoptosis following MMP disruption. To this end, DCFH-DA-based flow cytometric analysis was utilized to measure the amounts of ROS in control and sulforaphanetreated cells. As shown in Fig. 5A, compared with control cells, the production of ROS in sulforaphane-treated cells dramatically increased within $15 \mathrm{~min}$ of sulforaphane treatment and peaked at $30 \mathrm{~min}$ after treatment; thereafter, the production was attenuated gradually from 1 to $2 \mathrm{~h}$ following sulforaphane treatment. In a parallel experiment, pretreatment of the ROS scavenger NAC, along with sulforaphane, drastically decreased ROS generation as compared to the sulforaphanetreated group. Therefore, we proceeded to investigate whether ROS generation was necessary for sulforaphane-induced mitochondrial dysfunction. Fig. 5B shows that the scavenging of ROS by NAC significantly attenuated sulforaphane-induced mitochondrial membrane permeabilization. Furthermore, 

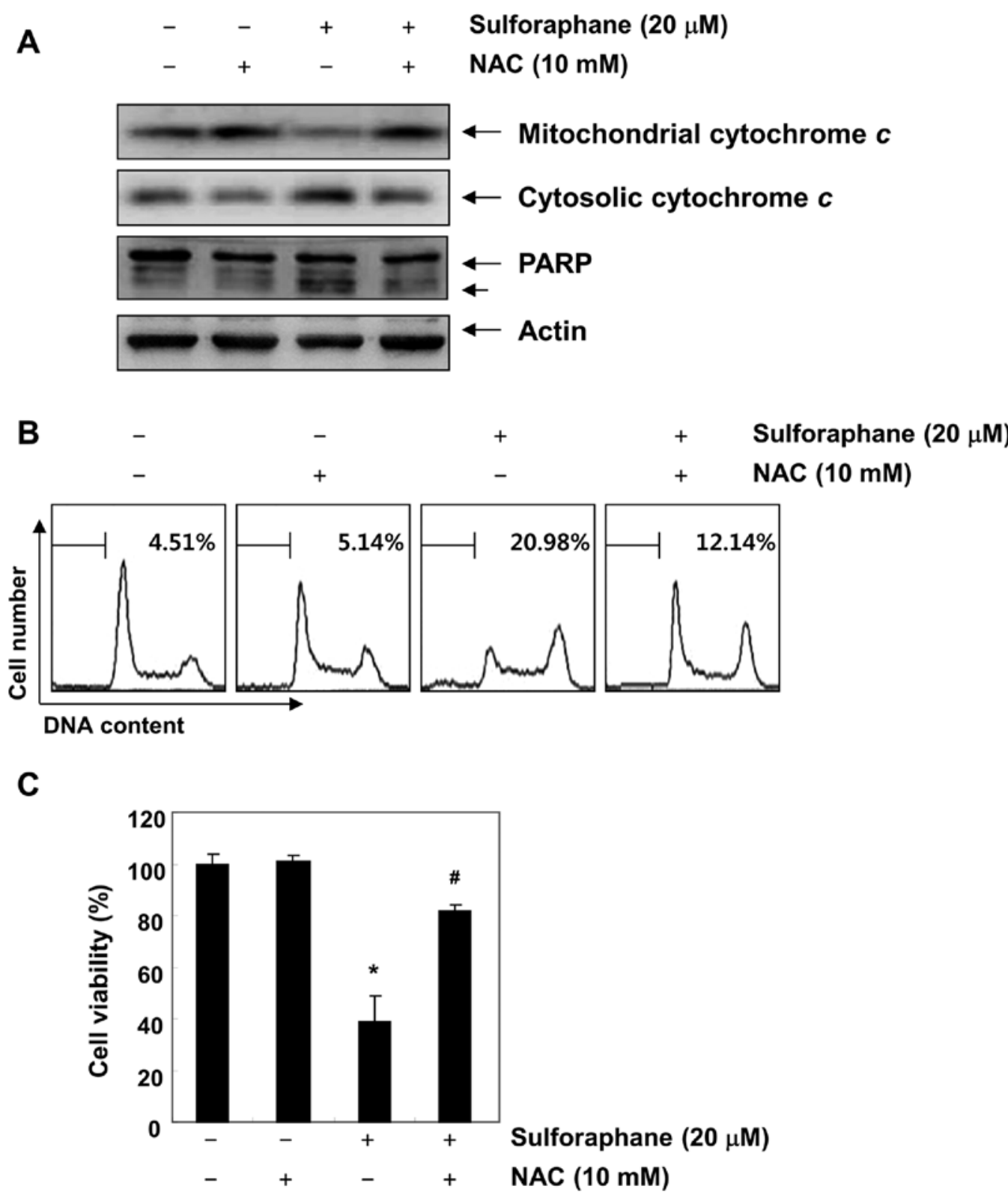

Figure 6. Induction of ROS-dependent apoptosis and growth inhibition by sulforaphane in T24 cells. Cells were pretreated with $10 \mathrm{mM} \mathrm{NAC} 1 \mathrm{~h}$ prior to $20 \mu \mathrm{M}$ sulforaphane treatment for $24 \mathrm{~h}$. (A) The cells were lysed, and the cellular proteins were then separated by SDS-polyacrylamide gels and transferred onto nitrocellulose membranes. The proteins were visualized using the indicated antibodies and an ECL detection system. Actin was used as an internal control. (B) Cells were stained with PI and were evaluated by a flow cytometer for sub-G1 DNA content, which represents the cells undergoing apoptotic DNA degradation. (C) Cell viability was analyzed using an MTT assay. The data are expressed as the mean \pm SD of triplicate samples. Significance was determined by Student's t-test ( ${ }^{*} \mathrm{p}<0.05$ vs. untreated control; ${ }^{\#} \mathrm{p}<0.05$ vs. sulforaphane-treated control).

blockade of ROS generation suppressed sulforaphane-induced cytochrome $c$ release, PARP cleavage, increases of sub-G1 accumulation, and growth inhibition (Fig. 6). These results indicate that ROS play a major role in sulforaphane-induced mitochondrial dysfunction in the T24 cell apoptosis process.

Sulforaphane upregulates the antioxidant response proteins Nrf2 and HO-1 in T24 cells. The transcription factor nuclear factor erythroid 2-related factor 2 (Nrf2) is one of the key regulators in the antioxidant response in eukaryotic cells. Under normal physiological conditions, the Nrf2 protein is sequestrated by its cytoplasmic partner, Kelch-like ECH-associated protein 1 (Keap1). ROS induce the release of Nrf2 from
Keap1 repression with a subsequent translocation of Nrf2 into the nucleus, where it binds to the antioxidant response element (ARE) for transcription of phase II detoxifying and antioxidant enzymes (29-31). In order to identify the mechanism underlying sulforaphane-induced apoptosis, we assessed the effect of sulforaphane on the Nrf2 pathway. As shown in Fig. 7A, an apparent increase in Nrf2 levels and a reduction in Keap1 protein expression were detected after treatment with sulforaphane. Next, Nrf2 activation was assessed by the accumulation of phosphorylated forms of the Nrf2 protein (p-Nrf2) in the nucleus, and we found that treatment with sulforaphane resulted in a translocation of $\mathrm{p}-\mathrm{Nrf} 2$ in the nucleus from cytosol (Fig. 7B). Because one of the downstream targets of $\mathrm{Nrf} 2$ is the 
A
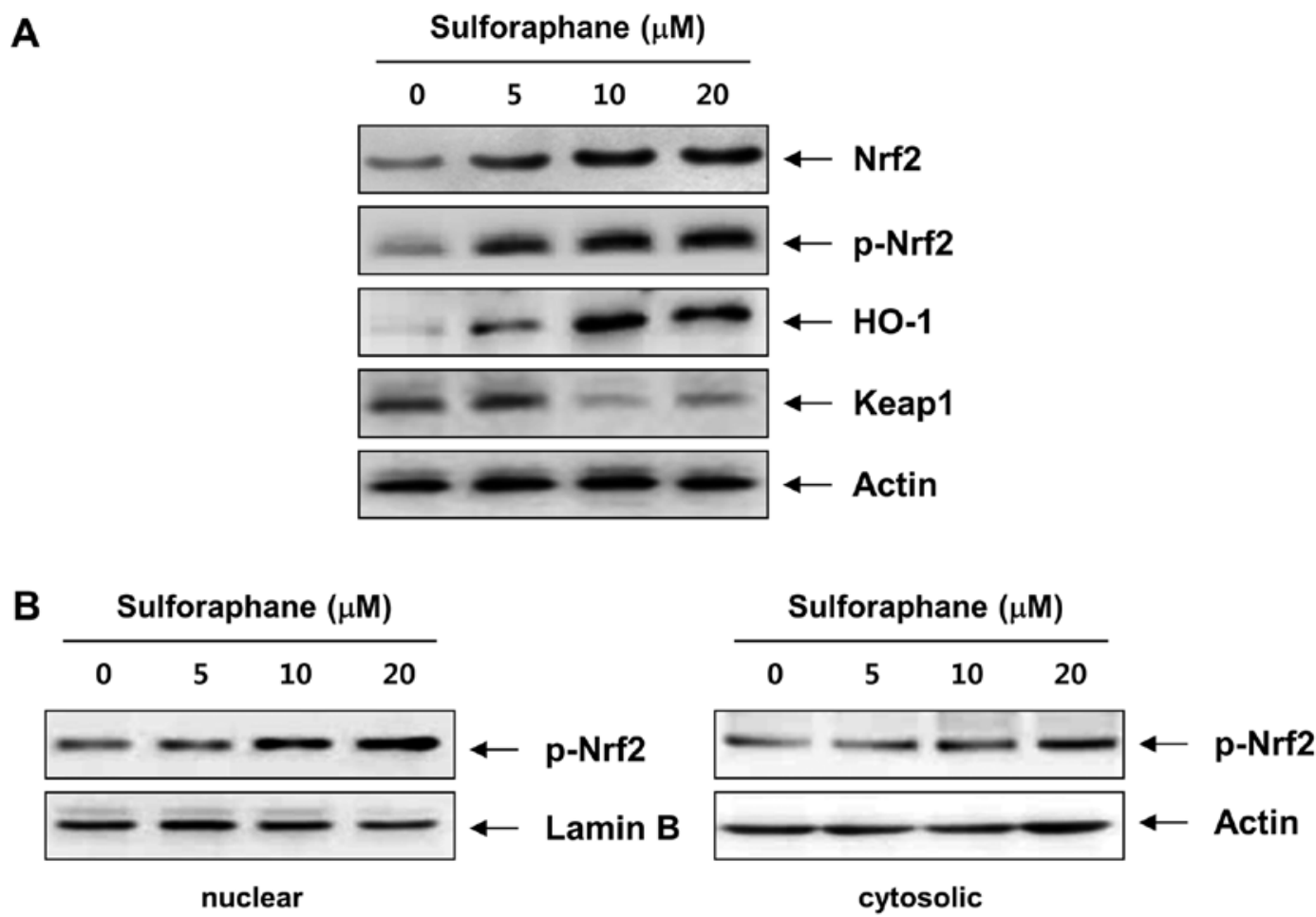

Figure 7. Effects of sulforaphane treatment on the levels of Nrf 2 and HO-1 in T2 4 cells. Cells were treated with $20 \mu \mathrm{M}$ sulforaphane for the indicated times. The cells were lysed, and equal amounts of total cellular (A), nuclear and cytosolic proteins (B) were then separated on SDS-polyacrylamide gels and transferred to nitrocellulose membranes. The membranes were probed with specific antibodies to total Nrf2, p-Nrf2, HO-1 and Keap1. The proteins were visualized using an ECL detection system. Lamin B and actin were used as internal controls for the nuclear and cytosolic fractions, respectively.

heme oxygenase-1 (HO-1) gene, whose gene product is a sensitive and reliable indicator of cellular stress $(32,33)$, in the next step, we evaluated the effect of sulforaphane on HO-1 protein levels following treatment of T24 cells. As illustrated in Fig. 7A, after treatment of T24 cells with sulforaphane, HO-1 levels increased steadily in a concentration-dependent manner, clearly indicating that sulforaphane treatment activated Nrf2 signaling in T24 cells.

\section{Discussion}

Because well-known natural phytochemicals extracted from plants have been used in an increasing number of cancer treatment applications, exploring plant-based anticancer agents has become an effective strategy for chemotherapeutic and anticancer drug development (12-14). In particular, epidemiological studies show that there is a low incidence of cancer among people who eat a lot of cruciferous vegetables. Sulforaphane is a phytochemical belonging to the family of isothiocyanates found in cruciferous vegetables, and is well known as a strong antioxidant and stimulator of natural detoxifying enzymes. Recently, the results of numerous experimental models $(34,35)$ have demonstrated the ability of sulforaphane to cause inhibition of cell growth and induction of apoptosis in cancer cells. Several studies have also demonstrated that sulforaphane can activate the extrinsic or intrinsic apoptotic pathways in a variety of cancer cell lines by altering the expression of apoptosisassociated or signaling proteins, cell cycle regulatory proteins, and transcription factors (15-17). Although sulforaphane may affect different signaling pathways depending on the cell type or culture conditions used, our previous results have shown that sulforaphane-induced apoptosis is correlated with the marked generation of intracellular ROS and loss of MMP, suggesting that this pro-oxidant function can play a pivotal role in sulforaphane-induced apoptosis in various human cancer cell lines (21,36-38). In accordance with these results, we observed a significant decrease in cell viability as well as an early increase in ROS levels after sulforaphane treatment (Fig. 5A). Moreover, the effects of sulforaphane on the depolarization of the MMP and apoptotic events were abrogated in the presence of NAC (Figs. 5B and 6). This result may reflect a chemotherapeutic potential of sulforaphane to induce oxidative injury in T24 cells. Furthermore, the apoptosis induced by the sulforaphane in T24 cells was related to the increase in the Bax/Bcl-2 ratio, translocation of Bax from cytosol to mitochondria, downregulation of IAP family proteins, release of cytochrome $c$ from the mitochondria, and activation of caspase- 9 and -3 , but not caspase- 8 (Figs. 3 and 4). These observations suggest that sulforaphane induces apoptosis in T24 cells via the ROS-mediated intrinsic pathway.

In addition to the ROS-mediated apoptosis induction, known cellular responses in sulforaphane-induced apoptosis include changes in the ER and Nrf2-ARE signaling pathways. ER is an important organelle involved in calcium signaling and the synthesis, folding and processing of proteins. Evidence is emerging that the impaired function of ER leads to ER stress, which is caused by oxidative stress, changes in $\mathrm{Ca}^{2+}$ homeostasis, and accumulation of unfolded or misfolded proteins, indicating that ER stress also plays a crucial role in the response to oxidative stress. The ER stress pathway is also another possible 
signaling pathway involved in anticancer agent-induced apoptosis in cancer cells $(27,28)$. A central regulator of ER function is GRP78, also referred to as a binding immunoglobulin protein (BiP), due to its roles in protein folding and assembly, targeting misfolded protein for degradation, ER $\mathrm{Ca}^{2+}$-binding and controlling the activation of transmembrane ER stress sensors. Due to its anti-apoptotic property, stress induction of GRP78 represents an important prosurvival component of the unfolded protein response $(39,40)$. In addition, one of the components of ER stress-mediated apoptosis is the transcription factor CHOP, also known as the growth arrest- and DNA damage-inducible gene 153 (GADD153), which is expressed at low levels under normal physiological conditions, but is strongly induced in response to ER stress. Subsequent upregulation of certain CHOP target genes promotes induction of ER stress-mediated apoptosis $(41,42)$. In the present study, we found that sulforaphane promoted the expression of GRP78 and CHOP (Fig. 3), suggesting that sulforaphane induced apoptosis in part through ER stress.

The Nrf2-ARE pathway is also involved in the modulation of oxidative and ER stresses and plays a cytoprotective role in response to these stresses. It is well known that the activation of the Nrf2-ARE pathway plays an important role in the antioxidant activity of sulforaphane $(42,43)$. Nrf2 is a transcription factor that binds to the cis-acting element in the genome, termed ARE in the regulatory regions of target genes. Under normal physiological conditions, Nrf2 is complexed with Keap1 in the cytoplasm and constantly degraded in the cytoplasm. However, upon exposure to oxidants and electrophiles, degradation of Nrf2 protein is halted, which makes it stabilized and phosphorylated, and free to translocate into the nucleus, thereby activating target phase II cytoprotective genes including HO-1 by binding to ARE (29,31). Moreover, ROS can induce the liberation of Nrf2 from Keap1 repression with a subsequent translocation of Nrf 2 into the nucleus. Furthermore, Nrf2 activation has been implicated in the promotion of cell survival following ER stress, and the ER stress signaling pathway can also activate an antioxidant program by preferentially inducing the expression of the mRNA encoding activating transcription factor 4 (ATF4) and by phosphorylation of Nrf2 $(44,45)$. In accordance with the previous data (46-52), we found activation of Nrf2-ARE signaling pathway by elevated levels of Nrf2 and HO-1, which was strongly correlated with an accumulation of phosphorylated Nrf2 in the nucleus and downregulation of Keap1 in sulforaphane-treated T24 cells (Fig. 7). Based on these observations, it is somewhat reasonable to conclude that Nrf2 activation mediates sulforaphane-induced apoptosis and ER stress in T24 cells. However, further studies are required to elucidate the function of Nrf2 target genes involved in the modulation of ER stress by sulforaphane.

Taken together, our findings demonstrate that sulforaphane induces apoptosis in T24 cells through the ROS-mediated intrinsic pathway, by increasing ROS production and inducing mitochondrial oxidative damage, MMP depolarization, and release of cytochrome $c$, as well as inducing an imbalance between Bax and Bcl-2, downregulation of IAP family proteins, activation of caspase- 9 and -3 , and the cleavage of PARP. Moreover, treatment with sulforaphane resulted in an increase of ER stress-associated proteins and accumulation of p-Nrf2 in the nucleus, indicating that sulforaphane-induced apoptosis is possibly related to the activation of the ER and Nrf2-ARE signaling pathways. However, the detailed mechanism by which sulforaphane affects the molecular connections between the two pathways remains unclear. Studies regarding this issue are ongoing.

\section{Acknowledgements}

This research was supported by Grants from the Globalization of Korean Foods R\&D Program (912001-1), funded by the Ministry of Food, Agriculture, Forestry and Fisheries and the National Research Foundation of Korea (NRF) Grant funded by the Korea government (2008-0062611), Republic of Korea.

\section{References}

1. Cheung G, Sahai A, Billia M, Dasgupta P and Khan MS: Recent advances in the diagnosis and treatment of bladder cancer. BMC Med 11: 13, 2013.

2. Siegel R, Naishadham D and Jemal A: Cancer statistics, 2013. CA Cancer J Clin 63: 11-30, 2013.

3. Pinto-Leite R, Arantes-Rodrigues R, Palmeira C, Colaço B, Lopes C, Colaço A, Costa C, da Silva VM, Oliveira P and Santos L: Everolimus combined with cisplatin has a potential role in treatment of urothelial bladder cancer. Biomed Pharmacother 67: 116-121, 2013.

4. Black PC and Dinney CP: Bladder cancer angiogenesis and metastasis-translation from murine model to clinical trial. Cancer Metastasis Rev 26: 623-634, 2007.

5. Chung KM and Yu SW: Interplay between autophagy and programmed cell death in mammalian neural stem cells. BMB Rep 46: 383-390, 2013.

6. Elmore S: Apoptosis: a review of programmed cell death. Toxicol Pathol 35: 495-516, 2007.

7. Jin Z and El-Deiry WS: Overview of cell death signaling pathways. Cancer Biol Ther 4: 139-163, 2005.

8. Burz C, Berindan-Neagoe I, Balacescu O and Irimie A: Apoptosis in cancer: key molecular signaling pathways and therapy targets. Acta Oncol 48: 811-821, 2009.

9. Fleury C, Mignotte B and Vayssière JL: Mitochondrial reactive oxygen species in cell death signaling. Biochimie 84: 131-141, 2002.

10. Fruehauf JP and Meyskens FL Jr: Reactive oxygen species: a breath of life or death? Clin Cancer Res 13: 789-794, 2007.

11. Saeidnia S and Abdollahi M: Toxicological and pharmacological concerns on oxidative stress and related diseases. Toxicol Appl Pharmacol 273: 442-455, 2013.

12. Dorai T and Aggarwal BB: Role of chemopreventive agents in cancer therapy. Cancer Lett 215: 129-140, 2004.

13. Shu L, Cheung KL, Khor TO, Chen C and Kong AN: Phytochemicals: cancer chemoprevention and suppression of tumor onset and metastasis. Cancer Metastasis Rev 29: 483-502, 2010.

14. Vinod BS, Maliekal TT and Anto RJ: Phytochemicals as chemosensitizers: from molecular mechanism to clinical significance. Antioxid Redox Signal 18: 1307-1348, 2013

15. Fimognari $C$ and Hrelia P: Sulforaphane as a promising molecule for fighting cancer. Mutat Res 635: 90-104, 2007.

16. Clarke JD, Dashwood RH and Ho E: Multi-targeted prevention of cancer by sulforaphane. Cancer Lett 269: 291-304, 2008.

17. Cheung KL and Kong AN: Molecular targets of dietary phenethyl isothiocyanate and sulforaphane for cancer chemoprevention. AAPS J 12: 87-97, 2010.

18. Shan Y, Wang X, Wang W, He C and Bao Y: p38 MAPK plays a distinct role in sulforaphane-induced up-regulation of ARE-dependent enzymes and down-regulation of COX-2 in human bladder cancer cells. Oncol Rep 23: 1133-1138, 2010.

19. Ding Y, Paonessa JD, Randall KL, Argoti D, Chen L, Vouros P and Zhang Y: Sulforaphane inhibits 4-aminobiphenyl-induced DNA damage in bladder cells and tissues. Carcinogenesis 31: 1999-2003, 2010

20. Shan Y, Zhang L, Bao Y, Li B, He C, Gao M, Feng X, Xu W Zhang $X$ and Wang S: Epithelial-mesenchymal transition, a novel target of sulforaphane via COX-2/MMP2, 9/Snail, ZEB1 and miR-200c/ZEB1 pathways in human bladder cancer cells. J Nutr Biochem 24: 1062-1069, 2013. 
21. Park HS, Han MH, Kim GY, Moon SK, Kim WJ, Hwang HJ, Park KY and Choi YH: Sulforaphane induces reactive oxygen species-mediated mitotic arrest and subsequent apoptosis in human bladder cancer 5637 cells. Food Chem Toxicol 64 157-165, 2014.

22. Kim NH, Hong BK, Choi SY, Moo Kwon H, Cho CS, Yi EC and Kim WU: Reactive oxygen species regulate context-dependent inhibition of NFAT5 target genes. Exp Mol Med 45: e32, 2013.

23. MacKenzie SH and Clark AC: Targeting cell death in tumors by activating caspases. Curr Cancer Drug Targets 8: 98-109, 2008.

24. Wen X, Lin ZQ, Liu B and Wei YQ: Caspase-mediated programmed cell death pathways as potential therapeutic targets in cancer. Cell Prolif 45: 217-224, 2012.

25. Susnow N, Zeng L, Margineantu D and Hockenbery DM: Bcl-2 family proteins as regulators of oxidative stress. Semin Cancer Biol 19: 42-49, 2009.

26. Ola MS, Nawaz M and Ahsan $\mathrm{H}$ : Role of Bcl-2 family proteins and caspases in the regulation of apoptosis. Mol Cell Biochem 351: 41-58, 2011.

27. Shore GC, Papa FR and Oakes SA: Signaling cell death from the endoplasmic reticulum stress response. Curr Opin Cell Biol 23 . 143-149, 2011.

28. Logue SE, Cleary P, Saveljeva S and Samali A: New directions in ER stress-induced cell death. Apoptosis 18: 537-546, 2013.

29. Li N and Nel AE: Role of the Nrf2-mediated signaling pathway as a negative regulator of inflammation: implications for the impact of particulate pollutants on asthma. Antioxid Redox Signal 8: 88-98, 2006

30. Keum YS: Regulation of the Keap1/Nrf2 system by chemopreventive sulforaphane: implications of posttranslational modifications. Ann N Y Acad Sci 1229: 184-189, 2011.

31. Kansanen E, Kuosmanen SM, Leinonen H and Levonen AL: The Keap1-Nrf2 pathway: mechanisms of activation and dysregulation in cancer. Redox Biol 1: 45-49, 2013.

32. Paine A, Eiz-Vesper B, Blasczyk R and Immenschuh S: Signaling to heme oxygenase-1 and its anti-inflammatory therapeutic potential. Biochem Pharmacol 80: 1895-1903, 2010.

33. Na HK and Surh YJ: Oncogenic potential of Nrf2 and its principal target protein heme oxygenase-1. Free Radic Biol Med 67C: 353-365, 2014

34. Roy SK, Srivastava RK and Shankar S: Inhibition of PI3K/AKT and MAPK/ERK pathways causes activation of FOXO transcription factor, leading to cell cycle arrest and apoptosis in pancreatic cancer. J Mol Signal 5: 10, 2010.

35. Bryant CS, Kumar S, Chamala S, Shah J, Pal J, Haider M, Seward S, Qazi AM, Morris R, Semaan A, Shammas MA, Steffes C, Potti RB, Prasad M, Weaver DW and Batchu RB Sulforaphane induces cell cycle arrest by protecting RB-E2F-1 complex in epithelial ovarian cancer cells. Mol Cancer 9: 47, 2010.

36. Choi WY, Choi BT, Lee WH and Choi YH: Sulforaphane generates reactive oxygen species leading to mitochondrial perturbation for apoptosis in human leukemia U937 cells. Biomed Pharmacother 62: 637-644, 2008

37. Moon DO, Kang SH, Kim KC, Kim MO, Choi YH and Kim GY: Sulforaphane decreases viability and telomerase activity in hepatocellular carcinoma Hep3B cells through the reactive oxygen species-dependent pathway. Cancer Lett 295: 260-266, 2010 .

38. Moon DO, Kim MO, Kang SH, Choi YH and Kim GY: Sulforaphane suppresses TNF-alpha-mediated activation of NF-kappaB and induces apoptosis through activation of reactive oxygen species-dependent caspase-3. Cancer Lett 274: 132-142, 2009.
39. Li J and Lee AS: Stress induction of GRP78/BiP and its role in cancer. Curr Mol Med 6: 45-54, 2006.

40. Roller C and Maddalo D: The molecular chaperone GRP78/BiP in the development of chemoresistance: mechanism and possible treatment. Front Pharmacol 4: 10, 2013.

41. Oyadomari S and Mori M: Roles of CHOP/GADD153 in endoplasmic reticulum stress. Cell Death Differ 11: 381-389, 2004

42. Sano R and Reed JC: ER stress-induced cell death mechanisms. Biochim Biophys Acta 1833: 3460-3470, 2013.

43. Cullinan SB and Diehl JA: Coordination of ER and oxidative stress signaling: the PERK/Nrf2 signaling pathway. Int J Biochem Cell Biol 38: 317-332, 2006.

44. Xu H, Zhou YL, Zhang XY, Lu P and Li GS: Activation of PERK signaling through fluoride-mediated endoplasmic reticulum stress in OS732 cells. Toxicology 277: 1-5, 2010.

45. Miyamoto N, Izumi H, Miyamoto R, Bin H, Kondo H, Tawara A, Sasaguri $\mathrm{Y}$ and Kohno K: Transcriptional regulation of activating transcription factor 4 under oxidative stress in retinal pigment epithelial ARPE-19/HPV-16 cells. Invest Ophthalmol Vis Sci 52: 1226-1234. 2011.

46. Jeong WS, Keum YS, Chen C, Jain MR, Shen G, Kim JH, Li W and Kong AN: Differential expression and stability of endogenous nuclear factor E2-related factor 2 (Nrf2) by natural chemopreventive compounds in HepG2 human hepatoma cells. J Biochem Mol Biol 38: 167-176, 2005.

47. Keum YS, Yu S, Chang PP, Yuan X, Kim JH, Xu C, Han J, Agarwal A and Kong AN: Mechanism of action of sulforaphane: inhibition of p38 mitogen-activated protein kinase isoforms contributing to the induction of antioxidant response elementmediated heme oxygenase-1 in human hepatoma HepG2 cells Cancer Res 66: 8804-8813, 2006.

48. Ping Z, Liu W, Kang Z, Cai J, Wang Q, Cheng N, Wang S, Wang S, Zhang JH and Sun X: Sulforaphane protects brains against hypoxic-ischemic injury through induction of Nrf2dependent phase 2 enzyme. Brain Res 1343: 178-185, 2010.

49. Lee YJ, Jeong HY, Kim YB, Lee YJ, Won SY, Shim JH, Cho MK, Nam HS and Lee SH: Reactive oxygen species and PI3K/Akt signaling play key roles in the induction of Nrf2-driven heme oxygenase-1 expression in sulforaphane-treated human mesothelioma MSTO-211H cells. Food Chem Toxicol 50: 116-123, 2012.

50. Oh CJ, Kim JY, Min AK, Park KG, Harris RA, Kim HJ and Lee IK: Sulforaphane attenuates hepatic fibrosis via NF-E2related factor 2 -mediated inhibition of transforming growth factor- $\beta /$ Smad signaling. Free Radic Biol Med 52: 671-682, 2012.

51. Alfieri A, Srivastava S, Siow RC, Cash D, Modo M, Duchen MR, Fraser PA, Williams SC and Mann GE: Sulforaphane preconditioning of the Nrf2/HO-1 defense pathway protects the cerebral vasculature against blood-brain barrier disruption and neurological deficits in stroke. Free Radic Biol Med 65: 1012-1022, 2013.

52. Kleszczyński K, Ernst IM, Wagner AE, Kruse N, Zillikens D, Rimbach G and Fischer TW: Sulforaphane and phenylethyl isothiocyanate protect human skin against UVR-induced oxidative stress and apoptosis: role of Nrf2-dependent gene expression and antioxidant enzymes. Pharmacol Res 78: 28-40, 2013. 